\title{
Probing subpicosecond dynamics using pulsed laser combined scanning tunneling microscopy
}

\author{
Osamu Takeuchi, Masahiro Aoyama, Ryuji Oshima, Yoshitaka Okada, Haruhiro Oigawa, \\ Nobuyuki Sano, and Hidemi Shigekawa ${ }^{\text {a) }}$ \\ Institute of Applied Physics, 21st COE, NANO Project, University of Tsukuba, Tsukuba 305-8573, Japan \\ Ryuji Morita and Mikio Yamashita \\ Department of Applied Physics, Hokkaido University, Sapporo 060-8628, Japan
}

(Received 10 May 2004; accepted 18 August 2004; publisher error corrected 5 November 2004)

\begin{abstract}
Time-resolved tunneling current measurement in the subpicosecond range was realized by ultrashort-pulse laser combined scanning tunneling microscopy, using the shaken-pulse-pair method. A low-temperature-grown $\mathrm{GaN}_{x} \mathrm{As}_{1-x}(x=0.36 \%)$ sample exhibited two ultrafast transient processes in the time-resolved tunnel current signal, whose lifetimes were determined to be $0.653 \pm 0.025$ and $55.1 \pm 5.0 \mathrm{ps}$. These values are of the same order of magnitude as those measured in the conventional pump-probe reflectivity measurement. () 2004 American Institute of Physics. [DOI: $10.1063 / 1.1804238]$
\end{abstract}

Smaller and faster are the key words in the progress of current nanoscience and technology. Thus, for further advances, a method of exploring the ultrafast transient dynamics of the local quantum functions in organized small structures is eagerly desired. Ultrashort optical pulse technology in the near-infrared to ultraviolet region has allowed us to observe transient phenomena in the femtosecond range, the optical-monocycle region, ${ }^{1,2}$ which, however, has a drawback of a relatively low spatial resolution due to electromagnetic wavelength. On the other hand, scanning tunneling microscopy (STM), although its time resolution is limited by circuit bandwidth $(\sim 100 \mathrm{kHz})$, enables us to observe spatial dynamics at the atomic level in real space. ${ }^{3}$ Therefore, the integration of ultrashort optical technology with STM has been one of the most exciting goals since their invention. ${ }^{4-7}$ Pioneering works were performed by Hamers et al..${ }^{4-7}$ which have attracted the extensive interest of researchers in various fields. However, there remain critical problems which have prevented the achievement of the laser-combined STM measurement, such as the displacement current due to the stray capacitance of the tunneling gap and photoelectrons produced by multiple photoabsorption. ${ }^{4-7}$ In such cases, since a large area is included in the processes, the superior space resolution of STM cannot be utilized. In particular, the thermal expansion of the STM tip by photoillumination causes much large noise in the tunneling current, making the measurement difficult.

Here, we show the results of the time-resolved tunneling current measurement in the subpicosecond range, which can advance the development of future research in terms of ultimate temporal and spatial resolutions.

A schematic of the measurement system is shown in Fig. 1 . We adopted the recently developed shaken-pulse-pairexcited STM (SPPX-STM) method, which realizes highly sensitive measurement free from the thermal expansion effect of the tip and sample. ${ }^{8}$ The tunneling junction is directly illuminated by a sequence of laser pulse pairs and average tunneling current, $\boldsymbol{I}_{t}\left(t_{d}\right)$, is measured as a function of the

\footnotetext{
a) Author to whom correspondence should be addressed; http:// dora.bk.tsukuba.ac.jp
}

delay time between the two pulses, $t_{d}$. To decrease broadband noise, the delay time of the two pulses $t_{d}$ is modulated with a small amplitude $\Delta t_{d}$ at frequency $\omega$, and the tunneling current is detected by a lock-in amplifier. Since the tunneling current $\boldsymbol{I}_{t}$ responds to the modulation as

$$
I_{t}\left(t_{d}+\Delta t_{d} \sin \omega t\right)=I_{t}\left(t_{d}\right)+\Delta t_{d} \sin \omega t \frac{d I_{t}}{d t_{d}}+O\left(\Delta t_{d}^{2}\right),
$$

the coefficient $\Delta t_{d}\left(d \boldsymbol{I}_{t} / d t_{d}\right)$ of the term sin $\omega t$ is obtained by lock-in detection. Since the laser intensity is not modulated in this system, thermal expansion and shrinking of the STM tip does not occur. Then, with the numerical integration of the $d \boldsymbol{I}_{t} / d t_{d}$ signal, $\boldsymbol{I}_{t}\left(t_{d}\right)$ can be obtained, conserving the spatial resolution of STM and high accuracy.

In the pulse-pair-excited STM measurement, the first laser pulse in each pulse pair acts as the pump pulse to excite and modulate the electronic structure of the sample surface, which might cause deviation in the tunneling current. Then, the tunnel current is again deviated by the second laser pulse. In the case of a short delay time between the two pulses, the excited state induced by the first pulse partially remains when the second pulse impinges on the sample. Thus, the

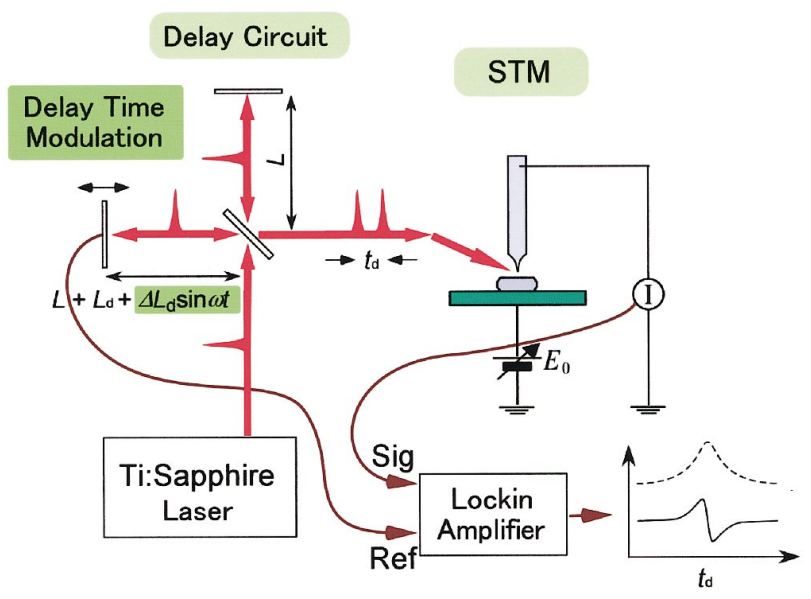

FIG. 1. (Color) Schematic of the system setup. 
amount of tunneling current deviation due to the second pulse can be different from that caused by the first pulse, and dependent on the delay time. Although such ultrashort spikes in the tunneling current cannot be resolved by any amplifier of present STM systems, if the deviation also changes the total amount of tunnel current for each pulse pair, this change can be detected as the average value of the tunneling current. Thus, we can observe the ultrafast transient phenomenon by measuring the time-averaged tunnel current, as the probe signal, as a function of the delay time.

In such measurement, the relationship between the excite/relax phenomena of interest and the time-resolved signal in the tunnel current is rather complex. However, this method combines and conserves the ultimate temporal resolution of the optical pump-probe approach and the ultimate spatial resolution of STM. Namely, SPPX-STM satisfies the requirement for exploring the ultrafast dynamics of the local quantum functions occurring in organized small structures.

Experiments were performed on low-temperature-grown $\mathrm{GaN}_{x} \mathrm{As}_{1-x}\left(x=0.36 \%\right.$; grown at $450{ }^{\circ} \mathrm{C}$ and annealed at $600{ }^{\circ} \mathrm{C}$ ), which is an excellent candidate for future fast information transfer systems and highly efficient solar cells, similar to other III-V-N compound semiconductors. ${ }^{9,10}$

For comparison, we first examined the dynamics of the $\mathrm{GaN}_{x} \mathrm{As}_{1-x}$ sample by the conventional optical pump-probe method. The system setup was similar to that shown in Fig. 1; Ti:sapphire excitation laser of $300 \mathrm{~mW}$, central wavelength of $800 \mathrm{~nm}$, bandwidth of $30 \mathrm{~nm}$, pulse width of $25 \mathrm{fs}$, and oscillation frequency of $80 \mathrm{MHz}$. The intensity of the pump pulses was adjusted to $\sim 5 \mathrm{~mW}$ focused into several micrometers. The polarizations of the pump and probe pulses were set perpendicular to each other. The sample was first excited by the intense pump pulse and the reflectivity just after pumping was measured by the probe pulse with six times lower intensity of the pump pulse. The intensity of the reflected probe pulse was measured as a function of the delay time between pumping and probing. In the spectrum, two exponential components with decay times of 0.8 and 10 ps were obtained. These were respectively attributed to the lifetimes of the intra- (faster one) and interband (slower one) carrier relaxation processes, similar to the case of GaAs. ${ }^{11,12}$

For the SPPX-STM measurement, performed in air, a weak feedback was applied to the STM $z$-piezo with a cutoff frequency less than $0.2 \mathrm{~Hz}$. The sample bias voltage and the reference current were set at $-2.0 \mathrm{~V}$ and $100 \mathrm{pA}$ in order to reduce the surface photovoltage effect by forming a forward bias condition. The incident angle of the laser pulses, $s$-polarized, was $40^{\circ}-50^{\circ}$, and the light intensity at the tipsample gap was $\sim 10 \mathrm{~mW}$ focused into $\sim 50 \mu \mathrm{m}$. The delay time was swept as a triangular function at $\sim 200$ s periodicity with a sinusoidal modulation of $0.5 \mathrm{ps}$ at $\sim 20 \mathrm{~Hz}$. The time constant of the lock-in-amplifier was $100 \mathrm{~ms}$ and the decay amount was $-24 \mathrm{~dB} /$ oct.

Figure 2(a) shows the raw signals and the fitting results as functions of delay time. The up and down scans represent the data obtained for the increasing and decreasing paths of the delay time, respectively. There is a slight shift of the two signals due to the existence of the lock-in time constant. The delay-time-dependent tunneling current, obtained by the numerical integration of the data in Fig. 2(a), is shown in Fig. 2(b). The vertical axis represents the amount of change in the tunneling current flowing from the tip to the sample. Here, the left-hand side of the graph is adjusted to the zero point (a)

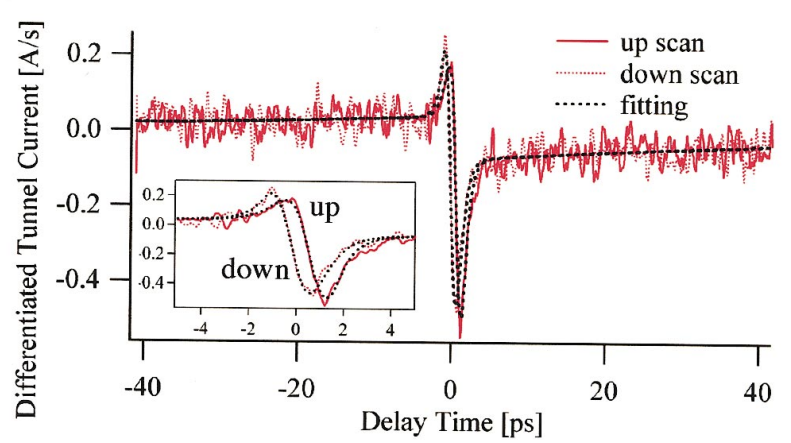

(b)

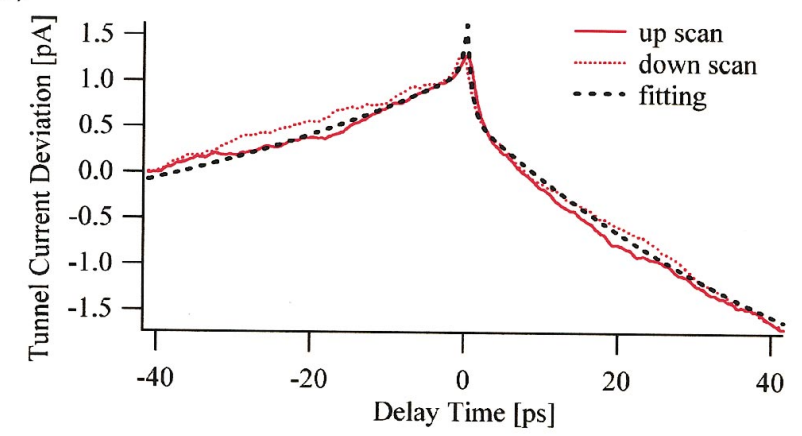

FIG. 2. (Color) (a) Derivative tunneling current as a function of delay time for a $\mathrm{GaN}_{x} \mathrm{As}_{1-x}(x=0.36 \%)$ sample annealed at $600{ }^{\circ} \mathrm{C}$. (b) Tunneling current obtained by numerical integration of the data in (a). The best-fit curve is drawn together.

for simplicity, since the absolute value cannot be determined by this experiment. There exist two exponential components with fast $(\sim 1.0 \mathrm{ps})$ and slow $(\sim 50 \mathrm{ps})$ decay times for each side of the $x$ axis. The curves for $t_{d}<0$ and $t_{d}>0$ were confirmed to have similar shapes at various intensity ratios, i.e., the time constant and the intensity ratio of the two decay components were always the same for both sides. The asymmetry of the graph for the negative and positive delay times is due to the difference between the light intensities of the two pulses (1:2 in this case; intense pulse impinging first for $t_{d}<0$ region). The difference between the baselines for the positive and negative sides indicates the existence of a longtime-scale physical process in the system, the origin of which is not yet clear.

The two decay times obtained by the SPPX-STM measurement are in similar ranges as those obtained by the conventional pump-probe reflectivity measurement, which suggests that the system is probing the same ultrafast transient phenomena. In the conventional optical pump-probe method, the density of the hot electrons excited by the first pulse is reflected in the change of the reflectivity of the second pulse, because the existence of the hot electrons prevents the following excitation of electrons into the same energy states. The change in the reflectivity observed by the optical pump-probe method, for a pump intensity two orders of magnitudes higher than that used in the SPPX-STM experiment, is on the order of $\Delta R / R=10^{-5}-10^{-6}$. Thus, the amount of change expected to appear in the average tunneling current upon the change in the reflectivity is obviously too small to be detected by the SPPX-STM measurement. Moreover, in the real measurement, a higher tunnel current was observed when the delay time was shorter despite the photoexcitation by the second pulse being smaller. Since the hot electrons 


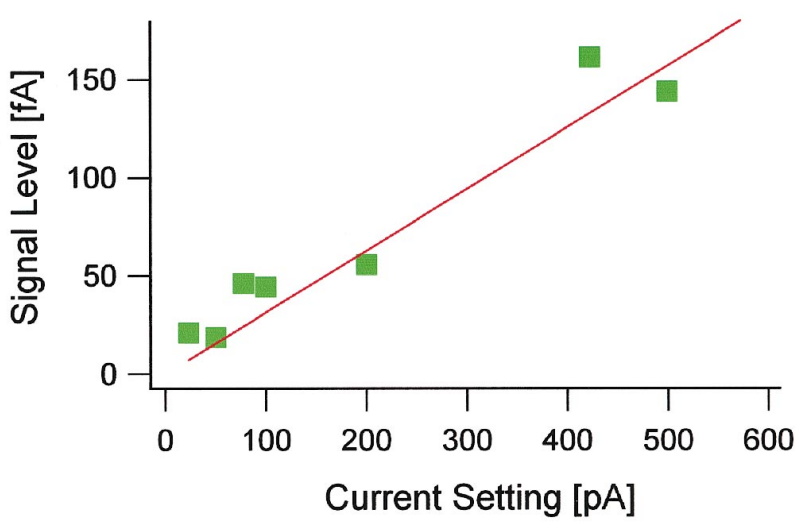

FIG. 3. (Color) Relationship between time-resolved signal intensity and average tunnel current, measured while varying tip-sample distance.

feel a lower tunneling barrier, the lower density of the hot electrons should result in a smaller tunnel current. Then, what causes the observed change in the average tunnel current in the SPPX-STM measurement?

In general, hot electrons produced by laser pulses kinetically relax first in energy space through energy-dissipating phonon scattering until they reach local thermal equilibrium, which takes place in the time scale of subpicoseconds to picoseoconds, depending on temperature (the kinetic stage). Then the hydrodynamic stage, relaxation processes from the local thermal equilibrium due to scatterings to the global equilibrium due to collective motion, follows with the time scale of a few tens of picoseconds. During the processes, the nonuniformity of the electron density induced locally by the laser pulse gradually vanishes. Finally, the overall thermal equilibrium is reached. ${ }^{13}$ When the electron density is high, the scenario is modified in some respects. Because of the small effective mass of GaAs, electrons easily degenerate as the electron density becomes higher. Since the local thermoequilibrium distribution function is given by the Fermi-Dirac distribution, the relaxation time in the kinetic stage of such hot electrons becomes longer, compared with the case of nondegeneracy, due to the Pauli exclusion principle. ${ }^{13} \mathrm{~A}$ rough estimation shows that the electron density excited by the pump and/or probe pulses in the present experiments exceeds well above $10^{18} \mathrm{~cm}^{-3}$, so that the electrons just after the excitation are strongly degenerate. The relaxation time in the kinetic stage of hot electrons excited by the second pulse could be, therefore, varied according to when the electrons are excited by the second pulse. In other words, as the delay time becomes shorter (the effect of degeneracy due to the first pulse is stronger), the relaxation time of hot electrons generated by the second pulse becomes longer and the deviation of the average tunneling current becomes greater. This scenario may explain the spectrum shown in Fig. 2.

In the development of the time-resolved STM, what is always questioned is whether the time-resolved signal is related to tunnel current or not, because this is the key issue which determines whether the method can have the superior spatial resolution of STM or not. ${ }^{4-8}$ Figure 3 shows the timeresolved signal intensity of SPPX-STM as a function of average tunnel current, which was measured while varying the tip-sample distance. There is a strong linear relationship. This indicates that the time-resolved signal is indeed ob- tained from the area just below the STM tip similar to ordinary STM measurement. Namely, the signal in Fig. 2 is the demonstration of subpicosecond time-resolved tunneling current measurement by laser-combined STM.

Before summarizing this study, we briefly mention the analytical procedure of the time-resolved signal obtained by SPPX-STM experiment. First, for extracting the lifetimes of the transient processes from a SPPX-STM spectrum, one should not directly compare the fitting functions (two exponential and one step function) and the integrated experimental data. This is due to the fact that the latter is strongly affected by the measurement conditions, such as the laser pulse width, amplitude of the delay time modulation, and the lock-in time constant. Thus, we must always take them into account in the fitting procedure. ${ }^{15}$ We found that the model function shown by the dotted line in Fig. 2(b) gives the best fit to the experimental result, the dotted lines in Fig. 2(a). The lifetimes, obtained in this analysis, are $0.653 \pm 0.025$ and $55.1 \pm 5.0 \mathrm{ps}$, respectively.

Second, when the delay time is as short as the pulse width, the interference between the two pulses causes the oscillation of the illumination intensity and breaks the condition of constant excitation intensity. ${ }^{7,8}$ This causes oscillation of tunnel current via the thermal expansion of the STM tip and/or other photoinduced current response. ${ }^{8,14}$ When the delay time modulation is larger than the pulse width, the oscillation causes an artifact signal in the region of $-\Delta t_{d}<t_{d}<\Delta t_{d}$. Thus, this signal can affect the observation of fast decay processes. This signal can, however, be effectively reduced by increasing $\Delta t_{d}$ and $\omega .{ }^{15}$ Actually, this component did not obviously appear in the present experimental result.

In conclusion, the time-resolved tunneling current measurement in the subpicosecond range was demonstrated, which can advance the development of future research in terms of ultimate temporal and spatial resolutions.

${ }^{1}$ A. Baltuska, Th. Udem, M. Uiberacker, M. Hentschel, E. Goulielmakis, CH. Gohle, R. Holzawarth, V. Yakovlev, A. Scrinzi, T. Hansch, and F. Krausz, Nature (London) 421, 611 (2003).

${ }^{2}$ K. Yamane, Z. Zhang, K. Oka, R. Morita, M. Yamashita, and A. Suguro, Opt. Lett. 28, 2258 (2003).

${ }^{3}$ Scanning Probe Microscopy and Spectroscopy, edited by R. Wiesendanger (Cambridge University Press, Cambridge, 1998).

${ }^{4}$ S. Weiss, D. Botkin, D. Ogletree, M. Salmeron, and D. Chemla, Phys. Status Solidi B 188, 343 (1995).

${ }^{5}$ S. Grafström, J. Appl. Phys. 91, 1717 (2002).

${ }^{6}$ R. Hamers and D. Cahill, Appl. Phys. Lett. 57, 2031 (1990).

${ }^{7}$ W. Pfeiffer, F. Sattler, S. Vogler, G. Gerber, J. Grand, and R. Moller, Appl. Phys. B: Lasers Opt. 64, 265 (1997).

${ }^{8}$ O. Takeuchi, R. Morita, M. Yamashita, and H. Shigekawa, Jpn. J. Appl. Phys., Part 1 41, 4994 (2002).

${ }^{9}$ S. R. Kurtz, J. F. Klem, A. A. Allerman, R. M. Sieg, C. H. Seager, and E. D. Jones, Appl. Phys. Lett. 80, 1379 (2002).

${ }^{10}$ A. Mintairov, T. Kosel, J. Merz, P. Blagnov, A. Vlasov, V. Ustinov, and R. Cook, Phys. Rev. Lett. 87, 277401 (2001).

${ }^{11}$ J. Yu, S. Horng, and C. Chi, Jpn. J. Appl. Phys., Part 1 37, 554 (1998).

${ }^{12}$ G. Segshneider, F. Jacob, T. Löffler, H. Roskos, S. Tautz, P. Kiesel, and G. Döhler, Phys. Rev. B 65, 125205 (2002).

${ }^{13}$ D. Zubarev, V. Morozov, and G. Ropke, Statistical Mechanics of Nonequilibrium Processes, Vol. 1 (Akademie, Berlin, 1996).

${ }^{14}$ O. Takeuchi, S. Yoshida, and H. Shigekawa, Appl. Phys. Lett. 84, 3645 (2004).

${ }^{15}$ Since space is limited, details about the signal analysis will be shown elsewhere. 